TRANSACTIONS OF THE

AMERICAN MATHEMATICAL SOCIETY

Volume 354, Number 4 , Pages 1583-1599

S 0002-9947(01)02888-4

Article electronically published on December 4, 2001

\title{
NONRADIAL SOLVABILITY STRUCTURE OF SUPER-DIFFUSIVE NONLINEAR PARABOLIC EQUATIONS
}

\author{
PANAGIOTA DASKALOPOULOS AND MANUEL DEL PINO
}

\begin{abstract}
We study the solvability of the Cauchy problem for the nonlinear parabolic equation

$$
\frac{\partial u}{\partial t}=\operatorname{div}\left(u^{m-1} \nabla u\right)
$$

when $m<0$ in $\mathbf{R}^{2}$, with $u(x, 0)=f(x)$ a given nonnegative function. It is known from earlier works of the authors that the asymptotic radial growth $r^{-2 / 1-m}, r=|x|$ for the spherical averages of $f(x)$ is critical for local solvability, in particular ensuring it if $f$ is radially symmetric. We show that if the initial data $f(x)$ behaves in polar coordinates like $r^{-2 / 1-m} g(\theta)$, for large $r=|x|$ with $g$ nonnegative and $2 \pi$-periodic, then the following holds: If $g$ vanishes on some interval of length $l^{*}=\frac{(m-1) \pi}{2 m}>0$, then there is no local solution of the initial value problem. On the other hand, if such an interval does not exist, then the initial value problem is locally solvable and the time of existence can be estimated explicitly.
\end{abstract}

\section{INTRODUCTION}

This paper studies the solvability of the Cauchy problem for the nonlinear parabolic equation

$$
\frac{\partial u}{\partial t}=\operatorname{div}\left(u^{m-1} \nabla u\right)
$$

in the range of exponents $m \leq 0$. We shall refer to (1.1) in this case as superdiffusive, in opposition to $m>1$ and $0<m<1$, called in standard terminology slow and fast diffusion respectively.

Equation (1.1) arises in a wide range of applications, out of which we note the spreading of microscopic droplets, proposed by López, Miller \& Ruckenstein [17] 1976, de Gennes [12] 1984, and Stratov [19] 1983. In a different context, Chayes, Osher and Ralston [4] have studied this equation in connection with interacting particle systems with self-organized criticality. When $m=0, N=2$, equation (1.1) arises in Geometry, as the two-dimensional Ricci flow, see Wu [22] and Hamilton [14.

Received by the editors March 25, 1998.

1991 Mathematics Subject Classification. Primary 35K15, 35K55, 35K65; Secondary 35J40.

The first author was partially supported by The Sloan Foundation and by NSF/Conicyt-Chile grant INT-9802406.

The second author was partially supported by grants Lineas Complementarias Fondecyt 8000010 and FONDAP. 
It is natural to ask under which conditions on $f$ the initial value problem

$$
\left\{\begin{aligned}
\frac{\partial u}{\partial t} & =\operatorname{div}\left(u^{m-1} \nabla u\right) \quad \text { in } \mathbf{R}^{N} \times(0, T), \\
u(x, 0) & =f(x), \quad x \in \mathbf{R}^{N},
\end{aligned}\right.
$$

with $T>0$ a given constant and $f$ a nonnegative, locally integrable function, is solvable. Writing equation (1.1) in the form

$$
\frac{\partial u}{\partial t}=\Delta \phi(u) \quad \text { in } Q_{T}
$$

where $\phi(u)=u^{m} / m$ if $m \neq 0,=\log u$ if $m=0$, and $Q_{T}=\mathbf{R}^{N} \times(0, T)$, we define the solution of (1.2) as a nonnegative function $u$ in $C\left([0, T) ; L_{\text {loc }}^{1}\left(\mathbf{R}^{N}\right)\right)$ such that $\phi(u)$ belongs to $L_{\text {loc }}^{1}\left(\mathbf{R}^{N} \times[0, T)\right)$ and which satisfies the equation in the distributional sense. Note that the assumption $\phi(u)$ locally integrable implies that $u$ is nonzero almost everywhere in $Q_{T}$.

It is proved in [20], that when $m \leq 0, N \geq 3$ or $m<0, N=2$, there exists no local solution to (1.2) if the initial data $f$ has finite mass, i.e. $f \in L^{1}\left(\mathbf{R}^{N}\right)$. In other words, the solution vanishes instantaneously because the high diffusion takes all mass to infinity in no time.

In the papers [7] and [8] the authors investigated the limiting decay rate marking the threshold between existence and nonexistence when $m \leq 0$. In fact, as it follows from the results in [7], the instant vanishing phenomenon occurs if

$$
\frac{1}{R^{N}} \int_{B_{R}} f(x) d x=o\left(R^{-2 /(1-m)}\right)
$$

as $R \rightarrow+\infty$. More precisely, when $m<0, N \geq 2$, it was shown in 8 that if

$$
\limsup _{R \rightarrow \infty} \frac{1}{R^{N-2 /(1-m)}} \int_{B_{R}} f(x) d x<C^{*} T^{1 /(1-m)}
$$

where

$$
C^{*}=\left[2\left(N-\frac{2}{1-m}\right)\right]^{1 /(1-m)} \omega_{N}
$$

with $\omega_{N}$ denoting the surface area of the unit sphere, then a solution to (1.2) must vanish identically before time $T$.

On the other hand, it was also shown in $[8]$ that if $f$ is radially symmetric and

$$
\liminf _{R \rightarrow \infty} \frac{1}{R^{N-2 /(1-m)}} \int_{B_{R}} f(x) d x \geq C^{*} T^{1 /(1-m)},
$$

then a solution to (1.2) defined up to time $T$ exists.

These results, based on integral comparisons with certain explicit solutions, show a notable symmetry between the super-diffusive case $m<0$ and the slow-diffusion or porous medium case $m>1$. In fact, from the theory for the porous medium equation developed in the papers [2], [3] and [6], it follows that if $m>1$ and

$$
\limsup _{R \rightarrow \infty} \frac{1}{R^{N-2 /(1-m)}} \int_{B_{R}} f(x) d x \leq C_{1} T^{1 /(1-m)} .
$$

then existence for (1.2) holds, while if

$$
\limsup _{R \rightarrow \infty} \frac{1}{R^{N-2 /(1-m)}} \int_{B_{R}} f(x) d x \geq C_{2} T^{1 /(1-m)},
$$


then a solution must blow-up before time $T$. Here $C_{1}$ and $C_{2}$ are constants dependent only on $m, N$. Let us observe that in the case of slow diffusion $m>1$, no radiality is assumed in the existence statement.

It should also be mentioned that for $m=0, N=2$ it is established in [9] (see also the independent works [21], 10]), that (1.2) is solvable (up to time $T$ ) if and only if $\int_{\mathbf{R}^{2}} f \geq 4 \pi T$ and no radiality is required for existence either.

It is thus tempting to guess that radial symmetry is not needed for a condition of the form (1.3) to guarantee existence. Strikingly enough, this is not true, as shown via an example in [8], when $m<0$ and $N=2$. Roughly speaking, it is shown that if $f$ vanishes on a "sufficiently wide" logarithmic spiral region, then no local solution exists. In such case the value of the limit in (1.3) can be arbitrarily large. A close look at the construction in 8 , shows that it can be simplified when $m \leq-1 / 3$, to yield that if $f$ vanishes on the infinite sector $0<\theta<l$ where $\theta$ is the polar angle and

$$
l>l^{*}=\frac{(m-1) \pi}{2 m}>0,
$$

then no local solution exists, i.e. (1.2) is not solvable for any $T>0$. Actually $l=l^{*}$ also yields nonexistence, or instant vanishing, as we will see.

These facts show that the super-diffusive equation may hide a rich and possibly very complex nonradial structure behind the existence question, not present in the slower diffussion case, where just the asymptotic behavior of the spherical averages of the initial data is the issue for solvability. Here the behavior of $f$ in the angle coordinate is also critical. It seems that when $m<0$, the fact that $f$ is too small in a "too large" region implies nonexistence, no matter what the behavior of $f$ outside that region is.

The reciprocal question remained unanswered: Assume that $N=2, m<0$. If $f$ is, say, the characteristic function of a sector with sufficiently wide aperture, is problem (1.2) solvable? The present paper is motivated by this question and we will show that its answer is affirmative. We will only consider the case of dimension $N=2$, since the problem becomes even more complex in higher dimensions. We will show that when $N=2, m<0$, the aperture $l^{*}$ defined above constitutes the exact threshold between existence of a globally defined solution and instant vanishing. We define, for $l>0$, the sector

$$
C_{l}=\{(r \cos \theta, r \sin \theta) \mid r>0,0<\theta<l\}
$$

and denote by $\chi_{l}$ its characteristic function, that is in polar coordinates,

$$
\chi_{l}(r, \theta)= \begin{cases}1 & \text { if } 0<\theta<l, \\ 0 & \text { otherwise }\end{cases}
$$

We define the critical length (or aperture) $l^{*}$ as

$$
l^{*}=\min \left\{2 \pi, \frac{(m-1)}{2 m} \pi\right\}>0 .
$$

Note that $l^{*}=2 \pi$ if and only if $m \geq-1 / 3$. The following result holds.

Theorem 1.1. Assume that $N=2, m<0$. (i) If $f \equiv 0$ a.e. on $C_{l^{*}}$, then the problem (1.2) is not locally solvable, i.e. (1.2) has no solution for any $T>0$.

(ii) Let $l<l^{*}$ and $f=\chi_{2 \pi-l}$. Then (1.2) admits a solution globally defined in time, that is for $T=+\infty$. 
The proof of the nonexistence statement (i) follows the lines of the counterexample developed in [8], but a refinement of the barriers constructed there is required in order to reach the exact aperture $l^{*}$.

Since, as we have seen, the asymptotic radial growth $r^{-2 / 1-m}$ for the spherical averages of $f(x)$ is also critical for local existence, it is natural to consider an initial data whose behavior for large $r=|x|$ is of the form $r^{-2 / 1-m} g(\theta)$, where $g$ is a nonnegative $2 \pi$-periodic function. It follows from the above result, that if $g$ vanishes on some interval of length $l^{*}$ then there is no local solution of problem (1.2). Rather surprisingly, if such an interval does not exist, then (1.2) for this $f$ is locally solvable, and the time of existence can be estimated explicitely. This will be shown in the next result.

To state this result in a precise way, we denote by $L_{2 \pi}^{\infty}$ the space of all bounded measurable $2 \pi$-periodic functions on the real line, and define for $0<l \leq 2 \pi$ and $0 \leq g \in L_{2 \pi}^{\infty}$ the number $K_{l}(g)$ as

$$
K_{l}(g)=\inf \left\{\int_{I} g(\theta) d \theta \mid I \text { interval, }|I|=l\right\} .
$$

Note that $K_{l}(g)$ is nondecreasing in $l$ and $K_{l}(g)=0$ if and only if there is an interval of length $l$ over which $g$ vanishes identically a.e.

Theorem 1.2. Assume that $N=2, m<0$. Let $f \in L_{l o c}^{1}\left(\mathbf{R}^{2}\right)$ which satisfies, for some $R>0$, that

$$
f(x) \geq r^{-2 /(1-m)} g(\theta), \quad \text { for } r=|x|>R
$$

where $g \in L_{2 \pi}^{\infty}$ is nonnegative. Assume that for some $0<l<l^{*}$ one has that $K_{l}(g)>0$. Then (1.2) has a local solution defined at least up to time $T$ given by

$$
T=\left(\frac{K_{l}(g)\left(l^{*}-l\right)^{4}}{C^{*}}\right)^{1-m} .
$$

where $C^{*}$ is a positive constant dependent only on $m$.

Note that combining Theorems 1.1 (a) and 1.2, we have that if $f(x)=r^{-2 /(1-m)} g(\theta)$, then (1.2) is locally solvable if and only if $K_{l^{*}}(g)>0$.

The rest of this paper will be devoted to the proofs of these results. Our approach is based on the relationship between local solvability of (1.2) and the existence of solutions for the elliptic problem

$$
\Delta v+v^{-\nu}=f(x) \quad \text { in } \mathbf{R}^{2},
$$

where $\nu=-1 / m>0$.

Indeed, we will prove in section 3 that if $u$ is a positive solution of (1.2), then the function

$$
\Phi(x)=\frac{1}{|m|} \int_{0}^{T} u^{m}(x, s) d s
$$

satisfies

$$
\Delta \Phi+(\gamma T)^{-1 / m} \Phi^{1 / m} \geq f
$$

with $\gamma=(1-m) /|m|$. Therefore solvability of (1.9) provides through (1.10) a barrier which prevents the possible vanishing of the maximal solution of (1.2). For 
the purpose of proving existence of solutions of (1.9), we will study first the special case of $f(x)$ of the form

$$
f(x)=r^{-2 \nu / 1+\nu} g(\theta),
$$

with $g \in L_{2 \pi}^{\infty}(\mathbf{R})$, nonnegative. An elementary but key observation is that in this case we can try a solution of the form

$$
v(x)=r^{2 / 1+\nu} w(\theta)
$$

where $w$ is $2 \pi$-periodic and solves the one-dimensional equation

$$
w_{\theta \theta}+\beta^{2} w+w^{-\nu}=g(\theta)
$$

where $\beta=\pi / l^{*}=2 /(\nu+1)$, since $\nu=-1 / m$. Our first task is to study solvability of this problem. We do this in $\S 2$. Our main result there asserts essentially that if $K_{l}(g)$ is sufficiently large, for some $l<l^{*}$, then the ordinary differential equation (1.11) possesses a $2 \pi$-periodic solution, a result that relies on a rather delicate construction of a $2 \pi$-periodic supersolution for this equation. In section 3 we extend this solvability result to the general elliptic problem and show the analogue of Theorem 1.2 for it. Section 4 is devoted to the proof of the parabolic results Theorems 1.2 and 1.1.

Finally, we would like to remark that a sufficient condition for solvability of (1.2) in the nonradial case has been given in [8], which roughly speaking asserts that if

$$
\int(\log |x-y|-\log |y|) f(y) \geq C(m) T^{1 / 1-m}|x|^{-2 m / 1-m},
$$

then (1.2) is solvable. The above integral is understood in a principal value sense by approximation with Green's functions on an expanding sequence of balls. This type of condition is of no use in the case of, say, the characteristic function of an infinite sector which we examine in this paper.

\section{2. $2 \pi$-PERIOdic Solutions of the O.D.E.}

In this section we will study the existence of $2 \pi$-periodic positive solutions of the one-dimensional nonlinear equation

$$
u^{\prime \prime}+\beta^{2} u+u^{-\nu}=g
$$

with $0 \leq g \in L_{2 \pi}^{\infty}$ and $\beta>0$. We denote in this section

$$
l^{*}=\min \left\{\frac{\pi}{\beta}, 2 \pi\right\}
$$

which recovers the $l^{*}$ in (1.5) when $\nu=-1 / m$ and $\beta=2 /(\nu+1)$.

For $l \in(0,2 \pi]$, we consider the number $K_{l}(g)$ as defined in (1.6). Our main result in this section states as follows.

Theorem 2.1. There exists a number $\lambda=\lambda(\nu, \beta)>0$, such that if for some $l \in(0,2 \pi]$ with $l<l^{*}$ we have

$$
K_{l}(g) \geq \frac{\lambda}{\left(l^{*}-l\right)^{4}}
$$

then equation (2.1) admits at least one $2 \pi$-periodic positive solution. 
The main step in the proof of this theorem will be the construction of a positive supersolution of the linear equation

$$
w^{\prime \prime}+\beta^{2} w=g
$$

when $0 \leq g \in L_{2 \pi}^{\infty}$ and $K_{l}(g)>0$ for some $l<l^{*}$. It should be remarked that the $2 \pi$-periodic solution of (2.3), which is unique if $\beta \neq n, n=1,2, \ldots$, does not need to be positive under this assumption.

Lemma 2.1. Assume that for some number $l<l^{*}$ one has $K_{l}(g)>0$. Then, there exists a $C^{1}, 2 \pi$-periodic supersolution $w$ of (2.3) such that

$$
\inf w \geq c(\beta) K_{l}(g)\left(l^{*}-l\right)^{4},
$$

for some constant $c(\beta)>0$.

First we present the proof of Theorem 2.1 based on the above lemma.

Proof of Theorem 2.1. Our proof is based on the sub-supersolution method. Since $g$ is bounded, the constant function $\underline{u}=\epsilon$, with $\epsilon$ arbitrarily small is a subsolution of the equation. Thus, it suffices to find a positive supersolution $\bar{u}$ of (2.1). Let us assume that $g$ satisfies (2.2) with $\lambda$ to be determined. Let $w$ be a supersolution of the linear equation (2.3) as in Lemma 2.1. We seek for a supersolution of the form

$$
\bar{u}=w-\gamma
$$

where $\gamma$ is an appropriately chosen positive constant. Then, $w$ satisfies

$$
w^{\prime \prime}+\beta^{2} w \leq g
$$

and

$$
\inf w \geq c(\beta) \lambda .
$$

A simple computation shows that the function $\bar{u}=w-\gamma$ is a supersolution of (2.1) if the constant $\gamma$ can be chosen such that $\bar{u}>0$ and

$$
-\beta^{2} \gamma+(w-\gamma)^{-\nu} \leq 0 .
$$

Setting $\gamma=\inf w / 2$ the above inequality is satisfied if

$$
\inf w \geq \frac{2}{\beta^{2 /(\nu+1)}} .
$$

Estimate (2.5) implies that this is possible if we choose

$$
\lambda=\frac{2}{c(\beta) \beta^{2 /(\nu+1)}} .
$$

Therefore $\bar{u}$ is the desired $2 \pi$-periodic supersolution. The existence of a $2 \pi$-periodic solution of (2.1) follows now from the standard sub-supersolution method.

Proof of Lemma 1.1. Let $g, l^{*}$ and $l$ be as in the statement of the lemma. With no loss of generality, we will assume in the rest of the proof that

$$
K_{l}(g)=1 .
$$

The idea is to construct a $2 \pi$-periodic function $h$, with $0 \leq h \leq g$, still satisfying $K_{l}(h)>0$, whose support is contained in a finite set of tiny intervals for which the construction of a supersolution $w$ of

$$
w^{\prime \prime}+\beta^{2} w=h
$$

with the desired properties will follow more directly. 
To this end we set

$$
\delta=\left(l^{*}-l\right) / N
$$

where $N \geq 10$ is a large integer to be determined in the course of the proof, in terms of $\beta$ only. Set $\mu=\delta /(2 l)$ and let $x_{0}$ be a point such that

$$
\int_{x_{0}}^{x_{0}+\delta} g \geq 2 \mu
$$

whose existence follows from condition (2.6).

We show next that there exists a partition of points $x_{0}<x_{1}<\ldots<x_{k}<x_{k+1}=$ $x_{0}+2 \pi$, such that for all $i=0,1, \ldots, k$

$$
\int_{x_{i}}^{x_{i}+\delta} g \geq \mu
$$

and also

$$
4 \delta<x_{i+1}-x_{i}<l+9 \delta .
$$

We have that $l<2 \pi$. Having chosen $\delta=\left(l^{*}-l\right) / N$ and $x_{0}$ satisfying (2.7) we take

$$
x_{1}=\sup \left\{x \in\left[x_{0}+\delta, x_{0}+l+5 \delta\right]: \int_{x}^{x+\delta} g \geq 2 \mu\right\} .
$$

Condition (2.6) assures that such a point exists and satisfies

$$
4 \delta \leq x_{1}-x_{0} \leq l+5 \delta .
$$

We proceed inductively. Assume that $x_{0}, \ldots, x_{j}$ have been chosen so that they satisfy (2.8) and (2.10). If $x_{j}+l+5 \delta<x_{0}+2 \pi$ we continue by choosing

$$
x_{j+1}=\sup \left\{x \in\left[x_{j}+\delta, x_{j}+l+5 \delta\right]: \int_{x}^{x+\delta} g d \theta \geq 2 \mu\right\}
$$

which, as before exists and satisfies

$$
4 \delta \leq x_{j+1}-x_{j} \leq l+5 \delta .
$$

If on the contrary $x_{0}+2 \pi-x_{j} \geq 5 \delta$, then in the case that $x_{j}+l+4 \delta \geq x_{0}+2 \pi$, we choose $x_{j}=x_{k}$ as the last point of our partition before $x_{0}+2 \pi$. If $x_{0}+2 \pi-x_{j} \leq 4 \delta$, then

$$
x_{0}+2 \pi-x_{j-1} \leq 4 \delta+\left(x_{j}-x_{j-1}\right) \leq l+9 \delta
$$

and therefore we can choose $x_{j-1}=x_{k}$ as the last point of our partition, still satisfying (2.9).

Then, in each of the intervals $I_{i}=\left[x_{i}, x_{i}+\delta\right]$ we choose a point $y_{i}$, which splits $I_{i}$ in such a way that on both intervals $I_{i}^{1}=\left[x_{i}, y_{i}\right]$ and $I_{i}^{2}=\left[y_{i}, x_{i}+\delta\right]$, one has

$$
\int_{I_{i}^{1}} g \geq \mu \quad \text { and } \quad \int_{I_{i}^{1}} g \geq \mu
$$

Next we consider a function $h$ on $\left[x_{0}, x_{0}+2 \pi\right]$ with $0 \leq h \leq g, h \equiv 0$ outside of the intervals $I_{i}$ and such that

$$
\int_{I_{i}^{1}} h=\int_{I_{i}^{2}} h=\mu
$$


We extend $h$ to the entire real line as a $2 \pi$-periodic function. Since $g$ is $2 \pi$-periodic we have

$$
0 \leq h \leq g .
$$

Therefore, the problem is reduced to constructing a supersolution $w$ of (2.3) with the right-hand side $h$. We will construct $w$ on the interval $\left[y_{0}, y_{0}+2 \pi\right]$ by solving the Neumann problems

$$
w^{\prime \prime}+\beta^{2} w=h_{i} ; \quad w^{\prime}\left(y_{i}\right)=w^{\prime}\left(y_{i+1}\right)=0,
$$

in each of the intervals $\left[y_{i}, y_{i+1}\right], i=0, \ldots, k$, where the functions $h_{i}$ will be chosen appropriately so that $0 \leq h_{i} \leq h$,

$$
w\left(y_{i}\right)=w\left(y_{i+1}\right), \quad \forall i
$$

and

$$
\inf w \geq c(\beta)\left(l^{*}-l\right)^{4}>0 .
$$

The construction of $w$ in each of the above intervals is based on the following lemma.

Lemma 2.2. There exists a positive integer $N=N(\beta)$ such that if $\delta$ and $s$ are positive numbers with

$$
3 \delta<s<l^{*}-N \delta
$$

and if $h$ is a nonnegative function on $[0, s]$ such that $h \equiv 0$ on $[\delta, s-\delta]$ and

$$
\int_{0}^{\delta} h=\int_{s-\delta}^{s} h=\mu
$$

then, there exists a function $w$ on $[0, s]$ which satisfies

$$
w^{\prime \prime}+\beta^{2} w \leq h ; \quad w^{\prime}(0)=w^{\prime}(s)=0,
$$

and in addition $w(0)=w(s)$ and

$$
\inf w \geq c(\beta) \mu \delta^{3} .
$$

Let us accept for now the validity of this fact, and let us finish the proof of Lemma 2.1. Each of the intervals $\left[y_{i}, y_{i+1}\right]$ has length $s_{i}$ satisfying

$$
3 \delta \leq s_{i} \leq l+9 \delta .
$$

It follows from Lemma 2.2 that there exists a positive integer $N=N(\beta)$ such that if we initially took $\delta=\left(l^{*}-l\right) / N$, then for every such interval there exists a $w_{i}$ satisfying

$$
w_{i}^{\prime \prime}+\beta^{2} w_{i} \leq h ; \quad w_{i}^{\prime}\left(y_{i}\right)=w_{i}^{\prime}\left(y_{i+1}\right)=0,
$$

with $w_{i}\left(y_{i}\right)=w_{i}\left(y_{i+1}\right)$ and

$$
\inf _{\left[y_{i}, y_{i+1}\right]} w_{i} \geq c(\beta)\left(l^{*}-l\right)^{4},
$$

(recall that $\mu=\delta / l$ ). Set

$$
\gamma=\min \left\{w_{i}\left(y_{i}\right) ; i=0,1, \ldots, k\right\}
$$

and

$$
\bar{w}_{i}=\gamma_{i}^{-1} w_{i},
$$


with $\gamma_{i}=w_{i}\left(y_{i}\right) / \gamma$ so that

$$
\bar{w}_{i}\left(y_{i}\right)=\gamma, \quad \forall i .
$$

Define $w$ on $\left[y_{0}, y_{0}+2 \pi\right]$ by setting $w=w_{i}$ on $\left[y_{i}, y_{i+1}\right]$ and extend it as a $2 \pi$ periodic function. It is clear that $w$ is the desired supersolution, which concludes the proof of the lemma, modulo the proof of Lemma 2.2.

Proof of Lemma 2.2. Denote by $G(x, y)$ the Green's function of the Neumann problem for the operator $L w=w^{\prime \prime}+\beta^{2} w$ on the interval $[0, s]$, so that

$$
G_{y y}(x, y)+\beta^{2} G(x, y)=\delta_{x}(y)
$$

$\delta_{x}(y)$ denoting the Dirac mass at $x$. An elementary computation shows that $G(x, y)$ is given by

$$
\frac{\cos (\beta(s-x)) \cos (\beta y)}{\beta \sin (\beta s)} \quad \text { if } 0<y<x
$$

and

$$
\frac{\cos (s x) \cos (\beta(y-s))}{\beta \sin (\beta s)} \text { if } y<x<s .
$$

We will show that there exists a positive $\lambda$ close to 1 , such that the solution $w$ of the Neumann problem for the equation $L w=h_{\lambda}$, with $h_{\lambda}=\lambda h$ on $[0, \delta]$ and $h_{\lambda}=h$ on $[s-\delta, s]$, satisfies both $w(0)=w(s)$ and (2.15). Since

$$
w(x)=\int_{0}^{s} G(x, y) h_{\lambda}(y) d y
$$

for the latter requirement we need

$$
w(0)=\lambda \int_{0}^{\delta} \frac{\cos (\beta(y-s))}{\beta \sin (\beta s)} h d y+\int_{s-\delta}^{s} \frac{\cos (\beta(y-s))}{\beta \sin (\beta s)} h d y
$$

to be equal to

$$
w(s)=\lambda \int_{0}^{\delta} \frac{\cos (\beta y)}{\beta \sin (\beta s)} h d y+\int_{s-\delta}^{s} \frac{\cos (\beta y)}{\beta \sin (\beta s)} h d y .
$$

In other words,

$$
\lambda \int_{0}^{\delta}[\cos (\beta y)-\cos (\beta(s-y))] h d y=\int_{s-\delta}^{s}[\cos (\beta(s-y))-\cos (\beta y)] h d y .
$$

From the mean value theorem

$$
\lambda=\frac{\cos \left(\beta \xi_{1}\right)-\cos \left(\beta\left(s-\xi_{1}\right)\right)}{\cos \left(\beta \xi_{2}\right)-\cos \left(\beta\left(s-\xi_{2}\right)\right)}
$$

for some $\xi_{1}, \xi_{2} \in[0, \delta]$, and thus we can estimate

$$
|\lambda-1| \leq \frac{\beta \sin (\beta s)}{1-\cos (\beta s)} \delta+c \delta^{2}
$$

where $c$ is a constant depending only on $\beta$. We will show next that $w$ satisfies (2.16). In the case that $\lambda<1$ the function $w$ will be the desired supersolution, otherwise we just replace $w$ by $w / \lambda$, to assure that (2.15) is satisfied. We will only estimate $w$ on $[0, s / 2]$, since the estimate on $[s / 2, s]$ will follow by symmetry. We have

$$
w(x)=\frac{\cos (\beta(s-x))}{\beta \sin (\beta s)} \int_{0}^{x} \cos (\beta y) h_{\lambda} d y+\frac{\cos (\beta x)}{\beta \sin (\beta s)} \int_{x}^{s} \cos (\beta(s-y)) h_{\lambda} d y
$$


and thus expanding $\cos (\beta(s-x))=\cos (\beta s) \cos (\beta x)+\sin (\beta s) \sin (\beta x)$ and dropping positive terms (remembering that $\delta$ is a small number and $0<\beta x<\pi / 2$ ), we conclude

$$
w(x) \geq \frac{\cos (\beta x)}{\beta \sin (\beta s)}\left\{\cos (\beta s) \lambda \int_{0}^{\delta} \cos (\beta y) h d y+\int_{s-\delta}^{s} \cos (\beta(s-y)) h d y\right\} .
$$

It remains to estimate the term $I$ inside brackets on the right-hand side of (2.18). If $\beta s \leq \pi / 2$, both terms in $I$ are nonnegative, and therefore dropping the first, we estimate

$$
I \geq \cos (\beta \delta) \int_{s-\delta}^{s} h d y \geq c(\beta) \mu
$$

which implies the estimate

$$
w(x) \geq \frac{\cos (\beta s / 2)}{\beta \sin (\beta s)} I \geq c(\beta) \mu .
$$

On the other hand, if $\pi / 2<\beta s<\beta l^{*}=\pi$, the two terms in $I$ have opposite sign and they compete. However, using (2.17) we estimate

$$
I \geq\left\{\cos (\beta s)\left[1+\beta \sin (\beta s) \delta+c \delta^{2}\right]+\cos (\beta \delta)\right\} \mu .
$$

We recall that also $\beta s<\beta l^{*}-N \beta \delta=\pi-N \beta \delta$. Let $\theta_{0}$ be a positive constant depending only on $\beta$ such that if $|\beta s-\pi|<\theta_{0}$ and $\beta \delta<\theta_{0}$

$$
\cos (\beta s) \geq-1+c(\pi-\beta s)^{2}, \quad \cos (\beta \delta) \geq 1-c \delta^{2}, \quad \cos (\beta s / 2) \geq c(\pi / 2-\beta s / 2)
$$

and

$$
(\pi-\beta s) / 2 \leq \sin (\beta s) \leq \pi-\beta s
$$

Then,

$$
I \geq\left(c_{1}(\pi-\beta s)^{2}-c_{2}(\pi-\beta s) \delta-c_{3} \delta^{2}\right) \mu
$$

with $c_{1}, c_{2}, c_{3}$ positive, depending only on $\beta$. Thus

$$
I \geq\left(\left(c_{1} N-c_{2}\right)(\pi-\beta s)-c_{3} \delta\right) \delta \mu .
$$

Hence if $N$ was large enough a priori, only in terms of $\beta$, we may conclude $I \geq$ $C(\beta) \delta^{2} \mu$, and hence

$$
w(x) \geq \frac{\cos (\beta x)}{\beta \sin (\beta s)} I \geq C(\beta) \delta^{3} \mu .
$$

Finally in the last case $\pi / 2<\beta s<\pi-\theta_{0}$, we have

$$
I \geq\left\{\cos \left(\pi-\theta_{0}\right)+\cos (\beta \delta)-c_{2} \delta-c_{3} \delta^{2}\right\} \mu
$$

and hence if $\delta$ is sufficiently small

$$
I \geq c(\beta) \mu
$$

which implies that

$$
w(x) \geq \frac{\cos (\beta s / 2)}{\beta \sin (\beta s)} I \geq c(\beta) \mu .
$$

The desired estimate (2.16) thus follows. 


\section{The Elliptic Equation}

In this section we will study the existence of solutions for the elliptic equation

$$
\Delta u+u^{-\nu}=f(x), \quad \text { in } \mathbf{R}^{2}
$$

where $f$ is a nonnegative, locally integrable function. As we observed in the introduction, in the special $f=r^{-2 \nu /(\nu+1)} g(\theta)$ one can set, in polar coordinates,

$$
u(r, \theta)=r^{2 /(\nu+1)} \tilde{u}(\theta),
$$

so that (3.1) reduces to the problem of finding positive, $2 \pi$-periodic solutions of the ordinary differential equation

$$
\tilde{u}^{\prime \prime}+\beta^{2} \tilde{u}+\tilde{u}^{-\nu}=g(\theta)
$$

with $\beta=2 /(\nu+1)$, an equation of the form studied in the previous section. As in there we set $l^{*}=\min \{2 \pi, \pi(\nu+1) / 2\}$. Then, $l^{*}=2 \pi$ iff $\nu \geq 3$.

Our main existence result for equation (3.1) states as follows.

Theorem 3.1. Assume that $N=2$ and $\nu>0$. Then there exists a constant $C^{*}=C^{*}(\nu)>0$ such that, if for some number $0<l<l^{*}$ one has

$$
f(x) \geq r^{-2 \nu /(\nu+1)} g(\theta), \quad \text { for }|x| \geq R
$$

with

$$
K_{l}(g) \geq \frac{C^{*}}{\left(l^{*}-l\right)^{4}}
$$

then equation (3.1) admits at least one positive solution.

If $f=r^{-2 \nu /(\nu+1)} g(\theta)$, the existence of a solution $u$ of the form (3.2) is an immediate consequence of Theorem 1.1. For a general $f$ satisfying (3.3), the result follows from the next comparison lemma.

Lemma 3.1. Assume that for some $\eta>1$ one has the existence of a solution $w$ of

$$
\Delta w+\eta w^{-\nu}=h(x), \quad \text { in } \mathbf{R}^{2}
$$

satisfying

$$
\lim _{|x| \rightarrow \infty} \frac{\log |x|}{w(x)}=0 .
$$

Let $f \in L_{l o c}^{1}\left(\mathbf{R}^{2}\right)$ be a function such that for some number $R>0$

$$
f(x) \geq h(x), \quad \text { for }|x|>R .
$$

Then, the equation

$$
\Delta u+u^{-\nu}=f(x), \quad \text { in } \mathbf{R}^{2}
$$

is solvable.

Before proving this fact, let us finish the proof of the theorem.

Proof of Theorem 3.1. From Lemma 3.1 and condition (3.2) it is enough to show that for a number $\eta>1$ the equation

$$
\Delta w+\eta w^{-\nu}=r^{-2 \nu /(\nu+1)} g(\theta)
$$


is solvable, provided that condition (3.4) holds true for an appropriate $C^{*}$. By a dilation, we can easily check that this equation is equivalent to

$$
\Delta w+w^{-\nu}=r^{-2 \nu /(\nu+1)} \bar{g}(\theta),
$$

with $\bar{g}=\eta^{-(\nu+2) /(\nu+1)} g$. Thus its solvability is immediate from Theorem 2.1, provided that $C^{*}$ is chosen slightly larger than the constant $\lambda$ appearing in (2.2), and $\eta$ is sufficiently close to 1 .

Proof of Lemma 3.1. We will construct a supersolution $v$ of equation (3.6). Let $z(x)$ denote the Newtonian potential of the compactly supported function $(h-f)_{+}$, that is

$$
z(x)=-\frac{1}{2 \pi} \int_{\mathbf{R}^{2}} \log |x-y|(h-f)_{+}(y) d y .
$$

We look for a supersolution in the form

$$
v(x)=w(x)+z(x)+M,
$$

where $w$ is a solution to (3.5) and $M$ is an appropriately chosen large constant. First observe that since $w$ satisfies condition (3.6) and

$$
z(x)=\left\{-\frac{1}{2 \pi} \int_{\mathbf{R}^{2}}(h-f)_{+} d y\right\} \log |x|+o(|x|), \quad \text { as }|x| \rightarrow \infty,
$$

we can make $v(x)>0$ for all $x$, by choosing $M$ sufficiently large. On the other hand we have

$$
\Delta v+v^{-\nu}=\Delta w-(h-f)_{+}+v^{-\nu} \leq-\eta w^{-\nu}+h-(h-f)_{+}+v^{-\nu},
$$

and hence

$$
\Delta v+v^{-\nu} \leq f+w^{-\nu}\left\{\left(1+\frac{z(x)+M}{w(x)}\right)^{-\nu}-\eta\right\} .
$$

But thanks to (3.6) and (3.8) we can make

$$
\left(1+\frac{z(x)+M}{w(x)}\right)^{-\nu}-\eta<0
$$

by choosing $M$ sufficiently large so that

$$
\frac{z(x)+M}{w(x)}>\eta^{-1 / \nu}-1
$$

is satisfied, yielding $v$ as the desired supersolution. Having found $v$, the existence of a solution of (3.7) is now standard. For an increasing sequence $R_{n} \uparrow \infty$, let $u_{R_{n}}>0$ be the solution to the Dirichlet problem

$$
\begin{cases}\Delta u+u^{-\nu}=f & \text { in } B_{R_{n}}, \\ u(x)=0, & x \in \partial B_{R_{n}}\end{cases}
$$

whose existence is by now standard, see [5]. The maximum principle implies that

$$
u_{R_{n}} \leq u_{R_{n+1}} \leq v, \quad \text { in } B_{R_{n}}
$$

for all $R_{n}$ and thus the limit

$$
u=\lim _{R_{n} \rightarrow \infty} u_{R_{n}}
$$

is a solution of equation (3.7), as desired. 


\section{The Parabolic Initial Value Problem}

In this section we will give the proofs of the Theorems 1.1 and 1.2 concerning the solvability of the parabolic problem (1.2) for $N=2, m<0$. We will first prove Theorem 1.2. As we mentioned in the introduction, we are able to connect the elliptic and the parabolic problems via the following result, key for our purposes.

Proposition 4.1. Let $u>0$ be a smooth solution of $\partial u / \partial t=\operatorname{div}\left(u^{m-1} \nabla u\right)$ in $\Omega \times[0, T), \Omega \subset \mathbf{R}^{\mathbf{2}}$, with $u(\cdot, 0)=f$ smooth. Assume in addition that $u$ satisfies the Aronson - Bénilan inequality

$$
u_{t} \leq \frac{1}{(1-m) t} u, \quad \text { for } 0<t<T .
$$

Let us set

$$
\Phi(x, t)=\frac{1}{|m|} \int_{0}^{t} u^{m}(x, s) d s .
$$

Then the function $\Phi$ satisfies the differential inequality

$$
\Delta \Phi+(\gamma t)^{-1 / m} \Phi^{1 / m} \geq f
$$

with $\gamma=(1-m) /|m|$.

Proof. First notice that the Aronson - Bénilan inequality implies that for $0<s \leq$ $t<T$, one has

$$
u(x, s) \geq u(x, t)(s / t)^{1 /(1-m)}
$$

and therefore if $\alpha=m /(1-m)$

$$
\Phi(x, t)=\frac{1}{|m|} \int_{0}^{t} u^{m}(x, s) d s \leq \frac{u^{m}(x, t)}{|m|} t^{-\alpha} \int_{0}^{t} s^{\alpha} d s=\frac{1-m}{|m|} t u^{m}(x, t) .
$$

On the other hand

$$
\Delta \Phi=\frac{1}{|m|} \int_{0}^{t} \Delta u^{m}(x, s) d s=-\int_{0}^{t} u_{t}(x, s) d s=f(x)-u(x, t)
$$

with $u(x, t)$ estimated by $(4.2)$ as

$$
u(x, t) \leq\left(\frac{|m|}{(1-m) t}\right)^{1 / m} \Phi^{1 / m}=(\gamma t)^{-1 / m} \Phi^{1 / m} .
$$

Combining (4.3) and (4.4), the differential inequality (4.2) follows.

We are now ready for the proof of Theorem 1.2.

Proof of Theorem 1.2. Denote

$$
h(x)=r^{-2 /(1-m)} g(\theta) .
$$

Choose a smooth regularization $f_{\epsilon}$, of the initial data $f$, such that $f_{\epsilon} \geq \epsilon$,

$$
f_{\epsilon}(x) \geq h(x) \quad \text { for }|x| \geq R,
$$

and $f_{\epsilon} \rightarrow f$ in $L_{l o c}^{1}$, as $\epsilon \rightarrow 0$. Let $u_{\epsilon}$ be the unique smooth solution of the initial value problem $\partial u / \partial t=\operatorname{div}\left(u^{m-1} \nabla u\right)$, in $\mathbf{R}^{2} \times[0, \infty)$ with initial data $u_{\epsilon}(\cdot, 0)=f_{\epsilon}$. The existence, uniqueness and regularity of $u_{\epsilon}$ follow from the results in [7] and the classical theory of nondegenerate quasilinear parabolic equations [15]. Moreover, each of the $u_{\epsilon}^{\prime} s$ satisfy the Aronson-Bénilan inequality (4.2). This follows from the 
same argument employed in [1] for the porous medium case. For a given number $T>0$, we define

$$
\Phi_{\epsilon}(x)=\frac{1}{|m|} \int_{0}^{T} u_{\epsilon}^{m}(x, s) d s, \quad x \in \mathbf{R}^{2} .
$$

The function $\Phi_{\epsilon}$ satisfies

$$
\Delta \Phi_{\epsilon}+(\gamma T)^{-1 / m} \Phi_{\epsilon}^{1 / m} \geq f_{\epsilon}
$$

with $\gamma=(1-m) /|m|$, as shown in Lemma 4.1. Thus, setting

$$
\Psi_{\epsilon}(x)=\Phi_{\epsilon}(\rho x)
$$

with $\rho=(\gamma T)^{1 /(2 m)}$ we can easily compute that

$$
\Delta \Psi_{\epsilon}+\Psi_{\epsilon}^{-\nu} \geq \bar{f}_{\epsilon}
$$

with $\nu=-1 / m$ and $\bar{f}_{\epsilon}(x)=\rho^{2} f_{\epsilon}(\rho x)$ satisfying

$$
\bar{f}_{\epsilon}(x) \geq \rho^{2} h(\rho x)=(\gamma T)^{-1 /(1-m)} r^{-2 /(1-m)} g(\theta),
$$

for $|x| \geq R$. Set $\bar{h}(x)=\rho^{2} h(\rho x)$ and select a number $\delta \in[1,2]$. Our elliptic result, Theorem 2.1, shows that the equation

$$
\Delta v+\delta v^{-\nu}=\bar{h}, \quad \text { on } \mathbf{R}^{2}
$$

admits a solution $v$, provided that

$$
(\gamma T)^{-1 /(1-m)} K_{l}(g) \geq \frac{C(\nu, \delta)}{\left(l^{*}-l\right)^{4}} .
$$

Since $\delta \in[1,2]$ we may choose the constant $C(\nu, \delta)$ to depend only on $\nu=-1 / m$. Therefore this condition is assured if

$$
T \leq\left(\frac{K_{l}(g)\left(l^{*}-l\right)^{4}}{C^{*}}\right)^{1-m}
$$

as stated in (1.8), with $C^{*}=C(\nu, \delta) \gamma^{1-m}$ depending only on $m$. Moreover, if $z_{\epsilon}$ denotes the Newtonian potential of $\left(\bar{h}-\bar{f}_{\epsilon}\right)_{+}$, it follows from the maximum principle and the proof of Lemma 3.1 that

$$
\Psi_{\epsilon}(x) \leq v(x)+z_{\epsilon}(x)+M, \quad \forall x \in \mathbf{R}^{2}
$$

if $M$ is chosen sufficiently large so that

$$
v(x)+z_{\epsilon}(x)+M>0 \text { and } \frac{z_{\epsilon}+M}{v(x)}>1-\delta^{-1 / \nu} .
$$

By choosing the regularizations $f_{\epsilon}$ appropriately, we can make $M$ independent of $\epsilon$ and

$$
v(x)-z_{\epsilon}(x)+M \leq V(x), \quad \forall x \in \mathbf{R}^{2}
$$

with $V(x)$ a locally bounded function. Then, since

$$
\Phi_{\epsilon}(x)=\frac{1}{|m|} \int_{0}^{T} u_{\epsilon}^{m}(x, s) d s \leq C(m) V\left(\rho^{-1} x\right), \quad \forall x \in \mathbf{R}^{2},
$$

$\Phi_{\epsilon}(x)$ will also be uniformly bounded. This fact implies that the sequence of functions $\left\{u_{\epsilon}\right\}$ is uniformly bounded from below by a positive constant, on each compact subset of $\mathbf{R}^{2} \times(0, T)$. Indeed, from (4.1) we have

$$
u_{\epsilon}^{m}(x, \tau) s^{m /(1-m)} \leq u_{\epsilon}^{m}(x, s) \tau^{m /(1-m)} \quad \text { for } \quad \tau \leq s \leq T,
$$


and therefore integrating in $s$ on the interval $[\tau, T]$ and using (4.8) we conclude

$$
u_{\epsilon}^{m}(x, \tau) \leq C(m) V\left(\rho^{-1} x\right)(T-\tau)^{-1 /(1-m)} \tau^{m /(1-m)} .
$$

Since $\left\{u_{\epsilon}\right\}$ is also bounded from above, it follows that $\left\{u_{\epsilon}\right\}$ is equicontinuous on compact subsets of $\mathbf{R}^{\mathbf{2}} \times(0, T)$ and therefore, there exists a subsequence still denoted by $\left\{u_{\epsilon}\right\}$ and a nonnegative function $u$ such that

$$
u_{\epsilon} \rightarrow u, \quad \text { as } \epsilon \rightarrow 0
$$

uniformly on compact subsets of $\mathbf{R}^{2} \times(0, T)$. Letting $\epsilon \rightarrow 0$ (4.8) we conclude that

$$
\int_{0}^{T} u^{m}(x, s) d s \leq C(m) V\left(\rho^{-1} x\right), \quad \forall x \in \mathbf{R}^{2} .
$$

It is now standard (see [8]) to verify that $u$ is a solution of the initial value problem $(1.2)$.

We conclude this paper with the proof of Theorem 1.1.

Proof of Theorem 1.1. We begin with the proof of the existence assertion (ii) which is an almost immediate consequence of Theorem 1.2. First notice that $1-\chi_{l}=$ $g(\theta)$, with $g \in L_{2 \pi}^{\infty}$ being the characteristic function of the interval $[l, 2 \pi]$ extended periodically outside $[0,2 \pi]$. Fix a number $\bar{l} \in\left(l, l^{*}\right)$. Then

$$
K_{l}(g)=\bar{l}-l>0
$$

since $l<\bar{l}$. For any positive integer $n$ set $R_{n}=n^{1-m}$ so that $f(x)=1-\chi_{l}$ satisfies

$$
f(x) \geq n r^{-2 /(1-m)} g(\theta) \quad \text { for } r \geq R_{n} .
$$

Therefore, according to Theorem 1.2 there exists a solution $u_{n}$ of problem (1.2) with initial data $f$ defined at least up to time

$$
T_{n}=n\left(\frac{K_{\bar{l}}(g)\left(l^{*}-l\right)^{4}}{C^{*}}\right)^{1-m} .
$$

Since, from the maximum principle the solution $u_{\epsilon}$ of the initial value problem $\partial u / \partial t=\operatorname{div}\left(u^{m-1} \nabla u\right)$, in $\mathbf{R}^{2} \times[0, \infty)$ with initial data $u_{\epsilon}(\cdot, 0)=f+\epsilon$, satisfies

$$
u_{\epsilon} \geq u_{n}, \quad \forall n,
$$

and $T_{n} \uparrow \infty$ it follows that the decreasing limit

$$
u=\lim _{\epsilon \rightarrow 0} u_{\epsilon}
$$

is the desired globally defined solution.

Finally, we will prove the nonexistence assertion in Theorem 1.1, part (i). The proof is based on the following lemma.

Lemma 4.1. Let $0<\nu<3, \lambda>0$ and

$$
D_{\nu}=\left\{(r \cos \theta, r \sin \theta) \mid 0<r<1,0<\theta<\frac{\nu+1}{2} \pi\right\} .
$$

Then there exists a function $v \in C^{2}\left(D_{\nu}\right) \cap C\left(\bar{D}_{\nu}\right)$ such that

$$
\Delta v+\lambda v^{-\nu}>0, \quad \forall x \in D_{\nu}, v=0 \text { on } \partial D_{\nu}
$$


and

$$
\lim _{r \rightarrow 0^{+}} r^{-2 / \nu+1} v(r, \theta)=+\infty
$$

for all $0<\theta<(\nu+1) \pi / 2$.

Proof. We choose $v(r, \theta)$ of the form

$$
v(r, \theta)=\delta r^{\frac{2}{\nu+1}} \sin \left(\frac{2}{\nu+1} \theta\right)\left(-\log \frac{r}{2}\right)^{\sigma} \eta(r)
$$

where $\delta$ and $\sigma$ are parameters to be adjusted, and $\eta$ is a smooth, positive cut-off function on $(0,1)$ such that $\eta(r)=1$ for $0<r<1 / 2$, and $\eta(1)=0$. A direct computation shows that if one chooses $0<\sigma<1 /(\nu+1)$, then for $\delta$ sufficiently small one has

$$
\Delta v+\lambda v^{-\nu}>C \delta^{-\nu} r^{-2 \nu / \nu+1}(-\log (r / 2))^{-\nu \sigma}>0, \quad \text { in } D_{\nu}
$$

where $C$ depends on $\eta, \lambda, \nu$ and $\sigma$. It can be easily checked that $v$ defined in this way satisfies condition (4.10) and the proof is thus concluded.

Proof of Theorem 1.1 (i). We argue by contradiction. Assume that for some $f \in$ $L^{\infty}\left(\mathbf{R}^{2}\right)$ which vanishes on $C_{l^{*}}$, problem (1.2) has a solution $u(x, t)$ for some $T>0$. For any $R>0$ we denote by $C_{l^{*}}^{R}$ the bounded subregion of $C_{l^{*}}$ consisting of all points $(r, \theta)$ with $r<R$. We will construct smooth, positive functions $u_{R}$ defined on $C_{l^{*}}^{R} \times(0, \infty)$ satisfying

$$
\frac{\partial u_{R}}{\partial t}-\Delta\left(\frac{u_{R}^{m}}{m}\right) \geq 0
$$

such that $u_{R}(x, t) \rightarrow \infty$, as $x \rightarrow \partial C_{l^{*}}^{R}$ for all $t>0$, and with the property that

$$
\lim _{R \rightarrow \infty} u_{R}(x, t)=0, \quad \forall x \in C_{l^{*}}, t>0 .
$$

Assume for the moment that we have found such functions. Fix $T>0$. The maximum principle then implies that $u \leq v_{R}$ in $C_{l^{*}}^{R} \times(0, T)$. It follows that $u$ must vanish identically on $C_{l^{*}}$. This is impossible since $u$ must be positive almost everywhere, a contradiction which proves the result.

It remains to find the $u_{R}$ 's. To do this, we use the previous lemma. Choose there $\nu=-1 / m, \lambda=-m /(1-m)$, and observe that $D_{\nu}=C_{l^{*}}^{1}$. Let $v(r, \theta)$ be as in the lemma and define

$$
u_{R}(r, \theta, t)=R^{-2 /(1-m)}(t+1)^{1 /(1-m)} v^{1 / m}(r / R, \theta, t) .
$$

A direct computation then shows the validity of (4.10). On the other hand, for fixed $(r, \theta, t)$ we have

$$
\lim _{R \rightarrow \infty} u_{R}(r, \theta, t)=(t+1)^{1 /(1-m)} r^{2 /(1-m)} \lim _{\rho \rightarrow 0}\left(\rho^{2 m /(1-m)} v(\rho, \theta, t)\right)^{1 / m}=0
$$

Therefore all conditions required on $u_{R}$ are satisfied, and the proof is complete.

\section{REFERENCES}

[1] Aronson, D.G., Bénilan P., Régularité des solutions de l'équation de milieux poreux dans $\mathbf{R}^{n}$, C.R. Acad. Sci. Paris, 288, 1979, pp 103-105. MR 82i:35090

[2] Aronson, D.G. and Caffarelli, L.A., The initial trace of a solution of the porous medium equation, Trans. Amer. Math. Soc., 280, 1983, pp. 351-366. MR 85c:35042

[3] Bénilan, P., Crandall, M.G. and Pierre, M., Solutions of the porous medium equation under optimal conditions on the initial values, Indiana Univ. Math.J., 33, 1984, pp. 51-87. MR 86b:35084 
[4] Chayes, J.T., Osher, S.J. and Ralston, J.V., On singular diffusion equations with applications to self-organized criticality, Comm. Pure Appl. Math. 46, 1993, pp. 1363-1377. MR 94i:35086

[5] Crandall, M.G., Rabinowitz, P.H., Tartar, L., On a Dirichlet problem with a singular nonlinearity, Comm. Partial Diff. Eq., 2, 1977, pp. 193-222. MR 55:856

[6] Dahlberg, B.E.G., Kenig, C.E., Non-negative solutions to fast diffusions, Rev. Mat. Iberoamericana, 4, 1988, pp. 11-29. MR 90i:35128

[7] Daskalopoulos, P., del Pino M.A., On fast diffusion nonlinear heat equations and a related singular elliptic problem, Indiana Univ. Math. J., 43, 1994, pp. 703-728. MR 95g:35078

[8] Daskalopoulos,P., del Pino M.A., On nonlinear parabolic equations of very fast diffusion, Arch. Rational Mech. Analysis, 137, 1997, pp. 363-380. MR 98d:35094

[9] Daskalopoulos,P., del Pino M.A., On a Singular Diffusion Equation, Comm. in Analysis and Geometry, Vol. 3, 1995, pp 523-542. MR 97b:35116

[10] Davis, S.H., DiBenedetto, E., Diller, D.,J., Some a priori estimates for a singular evolution equation arising in thin film dynamics, SIAM J. Math. Anal., 27, 1996, pp. 638-660. MR 97a:35095

[11] Esteban, J.R., Rodriguez, A., Vazquez, J.L., A nonlinear heat equation with singular diffusivity, Arch. Rational Mech. Analysis, 103, 1988, pp. 985-1039. MR 89h:35167

[12] de Gennes, P.G., Wetting: statics and dynamics, Reviews of Modern Physics, 57 No 3, 1985, pp. 827-863.

[13] de Gennes, P.G., Spreading laws for microscopic droplets, C.R. Acad. Sci., Paris II, 298, 1984, pp. 475-478.

[14] Hamilton, R., The Ricci flow on surfaces, Contemp. Math., 71, Amer. Math. Soc., Providence, RI, 1988, pp. 237-262. MR 89i:53029

[15] Ladyzenskaja, O.A., Solonnikov, V.A., and Ural'ceva, N.N., Linear and quasilinear equations of parabolic type, Translations of Mathematical Monogrphs, 23, 1967. MR 39:3159b

[16] Lonngren, K.E., Hirose, A., Expansion of an electron cloud, Phys. Lett. A, 59, 1976, pp 285-286.

[17] Lopez, J., Miller, C.A., Ruckenstein, E., Spreading kinetics of liquid drops on solids, J. Coll. Int. Sci. 56, 1976, pp 460-461.

[18] Rosenau, P., On a Fast and Super-Fast Diffusion, preprint.

[19] Stratov, V.M., Speading of droplets of nonvotiable liquids over flat solid surface, Coll. J. USSR 45, 1983, 1009-1014.

[20] Vázquez, J.L., Nonexistence of solutions for nonlinear heat equations of fast-diffusion type, J. Math. Pures Appl., 71, 1992, pp. 503-526. MR 93k:35133

[21] Vázquez, J.L., Esteban, J.R., Rodriguez, R., The fast diffusion with logarithmic nonlinearity and the evolution of conformal metrics in the plane, Advances in Diff. Equations, 1, 1996. MR 97b:35096

[22] Wu, L.-F., A new result for the porous medium equation derived from the Ricci flow, Bull. Amer. Math. Soc., 28, 1993, pp 90-94. MR 93f:58245

Department of Mathematics, University of California at Irvine, Irvine, California 92697

E-mail address: pdaskalo@math.uci.edu

Departamento de Ingeniería Matemática and Centro de Modelamiento Matemático, Universidad de Chile, Casilla 170 Correo 3, Santiago, Chile

E-mail address: delpino@dim.uchile.cl 